\title{
Supplementary feeding of cattle-yak in the cold season alters rumen microbes, volatile fatty acids, and expression of SGLT1 in the rumen epithelium
}

\author{
Yuzhu Sha ${ }^{1}$, Jiang Hu ${ }^{1}$, Bingang Shi ${ }^{1}$, Renqing Dingkao ${ }^{2}$, Jiqing Wang ${ }^{1}$, Shaobin $\mathbf{L i}^{1}$, Wei Zhang ${ }^{1}$, Yuzhu Luo ${ }^{1}$, \\ Xiu Liu ${ }^{\text {Corresp. } 1}$ \\ ${ }^{1}$ College of Animal Science and Technology / Gansu Key Laboratory of Herbivorous Animal Biotechnology, Gansu Agricultural University, Lanzhou, Gansu, \\ China \\ 2 Institute of Animal Husbandry Science of Gannan Prefecture, Hezuo, Gansu, China \\ Corresponding Author: Xiu Liu \\ Email address: liuxiu@gsau.edu.cn
}

Cattle-yak, a hybrid offspring of yak (Bos grunniens) and cattle (Bos taurus), inhabit the Qinghai-Tibet Plateau at an altitude of more than 3,000 $\mathrm{m}$ and obtain nutrients predominantly through grazing on natural pastures. Severe shortages of pasture in the cold season leads to reductions in the weight and disease resistance of grazing cattle-yak, which then affects their production performance. This study aimed to investigate the effect of supplementary feeding during the cold season on the rumen microbial community of cattle-yak. Six cattle-yak (bulls) were randomly divided into two groups - "grazing + supplementary feeding" $(\mathrm{G}+\mathrm{S})(n=3)$ and grazing $(\mathrm{G})(n=3)$ - and rumen microbial community structure (based on 16S rRNA sequencing), volatile fatty acids (VFAs), and ruminal epithelial sodium ion-dependent glucose transporter 1 (SGLT1) expression were assessed. There were significant differences in the flora of the two groups at various taxonomic classification levels. For example, Bacteroidetes, Rikenellaceae, and Rikenellaceae_RC9_gut_group were significantly higher in the $\mathrm{G}+\mathrm{S}$ group than in the $\mathrm{G}$ group $(P<0.05)$, while Firmicutes and Christensenellaceae_R-7_group were significantly lower in the $\mathrm{G}+\mathrm{S}$ group than in the $\mathrm{G}$ group $(P<0.05)$. Kyoto Encyclopedia of Genes and Genomes (KEGG) and Clusters of Orthologous Groups (COG) analyses revealed that functions related to carbohydrate metabolism and energy production were significantly enriched in the $\mathrm{G}+\mathrm{S}$ group $(P<0.05)$. In addition, the concentration of total VFAs, along with concentrations of acetate, propionate, and butyrate, were significantly higher in the $\mathrm{G}+\mathrm{S}$ group than in the $\mathrm{G}$ group $(P<0.05)$. Furthermore, $S G L T 1$ expression in ruminal epithelial tissue was significantly lower in the $\mathrm{G}+\mathrm{S}$ group $(P<0.01)$. Supplementary feeding of cattle-yak after grazing in the cold season altered the microbial community structure and VFA contents in the rumen of the animals, and decreased ruminal epithelial SGLT1 expression. This indicated that supplementary feeding after grazing aids rumen function, 
improves adaptability of cattle-yak to the harsh environment of the Qinghai-Tibet Plateau, and enhances ability of the animals to overwinter. 
1 Supplementary feeding of cattle-yak in the cold season alters

2 rumen microbes, volatile fatty acids, and expression of

3 SGLT1 in the rumen epithelium

4 Yuzhu Sha ${ }^{1}$, Jiang $\mathrm{Hu}^{1}$, Bingang Shi ${ }^{1}$, Renqing Dingkao ${ }^{2}$, Jiqing Wang ${ }^{1}$, Shaobin $\mathrm{Li}^{1}$, Wei

5 Zhang ${ }^{1}$, Yuzhu Luo ${ }^{1}$, Xiu Liu ${ }^{1}$

6

$7 \quad{ }^{1}$ College of Animal Science and Technology / Gansu Key Laboratory of Herbivorous Animal

8 Biotechnology, Gansu Agricultural University, Lanzhou, Gansu, China

$9 \quad{ }^{2}$ Institute of Animal Husbandry Science of Gannan Prefecture, Hezuo, Gansu, China

11 Corresponding Author:

12 Xiu Liu

13 Yingmen village, Lanzhou, Gansu, 730070, China

14 Email address: liuxiu@gsau.edu.cn

15

16

17 


\section{Abstract}

Cattle-yak, a hybrid offspring of yak (Bos grunniens) and cattle (Bos taurus), inhabit the Qinghai-Tibet Plateau at an altitude of more than 3,000 m and obtain nutrients predominantly through grazing on natural pastures. Severe shortages of pasture in the cold season leads to reductions in the weight and disease resistance of grazing cattle-yak, which then affects their production performance. This study aimed to investigate the effect of supplementary feeding during the cold season on the rumen microbial community of cattle-yak. Six cattle-yak (bulls) were randomly divided into two groups - "grazing + supplementary feeding” $(G+S)(n=3)$ and grazing $(\mathrm{G})(n=3)$ - and rumen microbial community structure (based on 16S rRNA sequencing), volatile fatty acids (VFAs), and ruminal epithelial sodium ion-dependent glucose transporter 1 (SGLT1) expression were assessed. There were significant differences in the flora of the two groups at various taxonomic classification levels. For example, Bacteroidetes, Rikenellaceae, and Rikenellaceae_RC9_gut_group were significantly higher in the $\mathrm{G}+\mathrm{S}$ group than in the G group $(P<0.05)$, while Firmicutes and Christensenellaceae_R-7_group were significantly lower in the $\mathrm{G}+\mathrm{S}$ group than in the $\mathrm{G}$ group $(P<0.05)$. Kyoto Encyclopedia of Genes and Genomes (KEGG) and Clusters of Orthologous Groups (COG) analyses revealed that functions related to carbohydrate metabolism and energy production were significantly enriched in the $\mathrm{G}+\mathrm{S}$ group $(P<0.05)$. In addition, the concentration of total VFAs, along with concentrations of acetate, propionate, and butyrate, were significantly higher in the $G+S$ group than in the $G$ group $(P<0.05)$. Furthermore, SGLT1 expression in ruminal epithelial tissue was significantly lower in the $\mathrm{G}+\mathrm{S}$ group $(P<0.01)$. Supplementary feeding of cattle-yak after grazing in the cold season altered the microbial community structure and VFA contents in the rumen of the animals, and decreased ruminal epithelial SGLT1 expression. This indicated that supplementary feeding after grazing aids rumen function, improves adaptability of cattle-yak to the harsh environment of the Qinghai-Tibet Plateau, and enhances ability of the animals to overwinter.

\section{Keywords: cattle-yak; cold season; supplementary feeding; rumen microbes; VFAs}

\section{Introduction}

The mammalian digestive system is colonized by complex microorganisms. In particular, the rumen contains numerous microorganisms, such as bacteria, fungi, archaea, and protozoa, which participate in plant cell-wall hydrolysis and digestion of fibrous materials in the rumen. These materials are converted into absorbable compounds, such as bacterial proteins and volatile fatty acids (VFAs) (Nagaraja et al., 2016; Yu et al., 2020). Among them, VFAs are the main energy source of ruminant hosts, providing $70-80 \%$ of the energy requirements (Russell and Rychlik, 2001). VFAs are predominantly absorbed through the rumen epithelium to the blood via VFA transporters (Yohe et al., 2019). The rumen epithelium, a unique niche for interactions between the host and microorganisms, not only represents a physical barrier to the contents of the lumen but also affects net utilization of nutrients throughout the body (Lin et al., 2019; Malmuthuge and Guan, 2017). Glucose in the rumen is decomposed into VFAs by commensal microorganisms, but some glucose is transported directly to the blood via sodium ion-dependent glucose transporter 1 (SGLT1) (Aschenbach et al., 2000a). In this way, the energy consumed in the rumen 
58

fermentation process is saved and the glucose is directly transported to the blood to supply energy for the body (Aschenbach et al., 2000b; Aschenbach et al., 2002). Many studies have demonstrated a symbiotic relationship between intestinal microflora and the host. For example, changes in diet and physiology of the host can result in changes in gut microbes (Sun et al., 2016).

The cattle-yak is a hybrid offspring of yak (Bos grunniens) and cattle (Bos taurus), and has obvious heterosis (Wiener et al., 2011). The cattle-yak provides herders with meat, fur, fuel (animal dung as fuel), and other factors, and is an important component of animal husbandry in the Qinghai-Tibet Plateau. Like the yak, the cattle-yak lives at high-altitude and mainly obtains nutrients by grazing on natural pastures. The unique geographic environment of the alpine steppe area of the Qinghai-Tibet Plateau means each year is divided into a cold season and a warm season, which correspond to the grass period and hay period (when the grassland vegetation changes), respectively (Liu et al., 2020). In the hay period, grazing livestock such as yak and cattle-yak can only obtain nutrients by consuming hay, which seriously affects their production performance and results in various problems such as weight loss and lack of disease resistance (Sun et al., 2015; Xin et al., 2011). Supplementary feeding of high-altitude mammals after grazing in the cold season can improve the physical condition, immunity, and fertility of the animals, allowing them to successfully survive winter (Jing et al., 2018; Jing et al., 2017). However, there are limited reports on changes in the rumen microbial community, rumen VFAs, and related gene expression in the rumen tissues of the plateau cattle-yak after supplementary feeding combined with grazing in the cold season. We hypothesized that after supplemental feeding of cattle-yak in the harsh environment of the cold season, the microbial community, fermentation product VFAs, and gene expression in the rumen epithelium would undergo a series of changes. This study aimed to test this hypothesis by comparing rumen microorganisms, VFAs, and SGLT1 gene expression in cattle-yak that grazed only (G) with those that were also fed supplementary feed $(\mathrm{G}+\mathrm{S})$ in the cold season.

\section{Materials \& Methods}

\section{Ethics approval and sampling}

All experimental protocols were approved by the Livestock Care Committee of Gansu Agricultural University (GAU-LC-2020-055), and the experiment was conducted with the consent of the herdsman (Jie Gazang). Six cattle-yak (bulls) were selected from the cattle-yak (Gannan yak $9 \times$ Jersey cattle ${ }^{x}$ ) of a single herder in Gannan Tibetan Autonomous Prefecture, Gansu Province (359 $\pm 3.58 \mathrm{~kg}$; 2-3 years old). In the same cowshed, the animals were randomly divided into a "grazing + supplementary feeding" group $(G+S ; n=3)$ and a grazing control group (G; $n=3$ ), separated by fences. The two groups grazed in the same pasture during the day. At 6 PM, cattle-yaks of the G+S group were given access to supplementary feed of grass (Highland barley grass and Oat grass) + highland barley (concentrate) in a ratio of 3:7 concentrate to forage. Supplementary feeding was for a period of five months (from November to March of the following year). After the supplementary feeding period ended, the cattle-yak were slaughtered. Rumen organs were removed immediately after slaughter and contents of the rumen abdominal 
98

99

100

101

102

103

104

105

106

107

108

109

110

111

112

113

114

115

116

sac were collected. Fifty milliliters of rumen contents were obtained from each cattle-yak, filtered through four layers of sterile gauze, divided into three sterile cryogenic tubes, and immediately stored in liquid nitrogen for subsequent 16S rRNA gene analysis and determination of VFA concentrations. In addition, rumen epithelial tissue samples were acquired by dissecting a small piece of rumen abdominal sac, quickly removing rumen contents by rinsing with phosphatebuffered saline (PBS), then separating the epithelial tissue with blunt scissors. The tissue was immediately placed in liquid nitrogen and stored for subsequent RNA extraction to assess SGLT1 expression.

\section{DNA extraction and high-throughput sequencing}

A MN NucleoSpin 96 Soil kit (Macherey-Nagel, Germany) was used to extract microbial DNA from the rumen content samples, and the DNA concentration and purity were detected by NanoPhotometer (N60, Germany). All DNA samples were stored at $-80^{\circ} \mathrm{C}$ until subsequent processing. For analysis of the community structure of the rumen microorganisms, theV3-V4 region of the 16S rRNA gene was amplified from the DNA sample by PCR using universal primers 338F (5'-ACTCCTACGGGAGGCAGCAG-3') and 806R (5'GGACTACHVGGGTWTCTAAT-3'). The PCR comprised a pre-denaturation step at $95^{\circ} \mathrm{C}$ for 3 min, 40 cycles of denaturation at $95^{\circ} \mathrm{C}$ for $30 \mathrm{~s}$, annealing at $55^{\circ} \mathrm{C}$ for $30 \mathrm{~s}$, and extension at $72^{\circ} \mathrm{C}$ for $30 \mathrm{~s}$, followed by a final extension at $72^{\circ} \mathrm{C}$ for $7 \mathrm{~min}$. The library obtained by this PCR amplification process was sequenced on an Illumina MiSeq platform (Illumina, San Diego, CA, USA), and bioinformatics analysis was performed using BMKCloud (www.biocloud.net).

\section{Determination of rumen VFAs}

Concentrations of VFAs were determined using a gas chromatograph (GC-2010 Plus; Shimadzu, Kyoto, Japan) with an AT-free fatty acid phase (FFAP) chromatographic column (30 m $\times 0.32$ $\mathrm{mm} \times 0.25 \mathrm{~m}$ ). The internal standard method was employed using 2-ethyl butyric acid (2EB) as the internal standard. The detector temperature of the chromatograph was $260^{\circ} \mathrm{C}$, the temperature of the injection port was $250^{\circ} \mathrm{C}$, and the chromatographic column heating procedure comprised maintenance of temperature at $60^{\circ} \mathrm{C}$ for $1 \mathrm{~min}$, increase to $115^{\circ} \mathrm{C}$ at $5^{\circ} \mathrm{C} / \mathrm{min}$ (without maintenance), and then increase to $180^{\circ} \mathrm{C}$ at $15^{\circ} \mathrm{C} / \mathrm{min}$.

\section{mRNA expression analysis}

RNA isolater reagent (Vazyme, Nanjing, China, 7E371D9) was used to extract RNA from ruminal epithelial tissues. Concentration and purity of the extracted RNA were measured using an ultra-micro spectrophotometer (NanoDrop 2000; Thermo Scientific). cDNA synthesis was performed using a reverse transcription kit containing gDNA wiper (R323-01). Primer 5.0 software was used to design the gene primers (Table 1). A Q6 real-time fluorescence quantitative PCR system (Applied Biosystems) was used to quantify expression of SGLT1 and the internal reference gene ( $\beta$-actin). The reaction conditions comprised a pre-denaturation step at $95^{\circ} \mathrm{C}$ for $30 \mathrm{~s}$, followed by 40 cycles of $95^{\circ} \mathrm{C}$ for $10 \mathrm{~s}$ and $60^{\circ} \mathrm{C}$ for $30 \mathrm{~s}$. The dissolution curve analysis conditions were $95^{\circ} \mathrm{C}$ for $15 \mathrm{~s}, 60^{\circ} \mathrm{C}$ for $60 \mathrm{~s}$, and $95^{\circ} \mathrm{C}$ for $15 \mathrm{~s}$. The $20-\mu l$ reaction system contained 2×ChamQ Universal SYBR qPCR Master Mix (Q711-02), the cDNA template, and the

Peer) reviewing PDF | (2020:08:52441:2:0:NEW 1 Feb 2021) 
137 upstream and downstream primers. The data analysis was performed using the 2- $\Delta \Delta \mathrm{CT}$ method

138 (Livak and Schmittgen, 2001).

\section{Bioinformatics analysis}

140 Raw data returned by the Illumina MiSeq platform were subjected to merging of paired-end reads 141 (FLASH v1.2.7), filtering (Trimmomatic v0.33), and removal of chimeras (UCHIME v4.2) to 142 obtain optimized sequences (tags). UCLUST in QIIME (version 1.8.0) software was used to 143 cluster tags and obtain operational taxonomic units (OTUs) at the 97\% similarity level. OTUs 144 were annotated based on the SILVA bacterial taxonomy database. Taxonomic analysis was 145 subsequently performed at different taxonomic levels (phylum, class, order, family, and genus) to 146 obtain community structure maps and species clustering heatmaps. Species diversity within taxa 147 was analyzed using the $\alpha$-diversity indices ACE, Chao1, Shannon, and Simpson, and rarefaction 148 curves were plotted (Wang et al., 2012). $\beta$-diversity analysis was employed to compare 149 differences in species diversity (microbial composition and community structure) between 150 groups. PICRUSt software was used to predict metagenomic functional profiles of the microbial 151 communities in each group (identified by 16S rRNA gene sequencing) based on both the Kyoto 152 Encyclopedia of Genes and Genomes (KEGG) and Clusters of Orthologous Groups (COG) 153 databases.

\section{Statistical analysis}

155

Significance analysis of between-group differences (linear discriminant analysis effect size [LefSe] analysis) was used to explore potential biomarkers with statistical differences between the two groups (Segata et al., 2011). Metastats software was used to perform $t$-tests on the between-group differences in species relative abundance (White et al., 2009) and obtain $P$ values, which were corrected to obtain q values. Species that exhibited significant differences in relative abundance between the two groups (according to the q values) could then be identified. SPSS software v24.0 (SPSS, Inc., Chicago, Illinois) was used to analyze the data. Independent-sample $t$-tests were used to analyze the differences in rumen VFAs and SGLT1 expression between the $\mathrm{G}+\mathrm{S}$ and $\mathrm{G}$ groups in the cold seasons. Spearman correlation analysis was used to analyze the correlation between VFA levels and SGLT1 expression. $P$ values of 0.05 (two-tailed) and 0.01 (two-tailed) indicated significant differences, respectively.

\section{Results}

\section{S rRNA sequencing and rumen bacterial diversity}

168 A total of 160,213 pairs of reads were obtained by 16S rRNA gene sequencing (paired-end), and 169 127,677 clean tags were generated after merging of paired-end reads and filtering. Each sample generated at least 63,772 clean tags and a mean of 63,839 clean tags (with a mean sequence length of $412 \mathrm{bp}$ ). Tags were clustered at a similarity level of 97\% using USEARCH software and 502 OTUs were obtained. There were 498 OTUs in the G+S group and 499 in the G group, with 3 and 4 unique OTUs in the G+S and G groups, respectively (Fig. S1A). Rarefaction curves (Fig. S1B) indicate the species diversity and species richness in both groups. Limited new OTUs 
176 sufficient. There were no significant differences in the $\alpha$-diversity indices between the two groups $177 \quad(P>0.05)$ (Table 2).

\section{Microbial community structure in the rumen}

179 Eleven phyla were detected (Data S1), with Firmicutes, Bacteroidetes, and Actinobacteria 180 dominating both groups. The sum of the relative abundances of Firmicutes and Bacteroidetes 181 accounted for $>94 \%$ of all taxa in both groups. The relative abundance of Firmicutes was 182 significantly lower in the $\mathrm{G}+\mathrm{S}$ group than in the $\mathrm{G}$ group $(P<0.05)$, but the relative abundance of

183 Bacteroidetes was significantly higher $(P<0.05)$ (Fig. 1A). In addition, the 184 Firmicutes/Bacteroidetes ratio was significantly lower in the $G+S$ group (1.2035) than in the $G$ 185 group (4.3650; $P<0.05$ ). At the family level (Fig. 1B), Christensenellaceae and Ruminococcaceae 186 were the dominant families, and the relative abundance of Rikenellaceae was significantly higher 187 in the $\mathrm{G}+\mathrm{S}$ group than in the $\mathrm{G}$ group $(P<0.05)$. At the genus level (Fig. 1C), 123 bacterial taxa 188 were detected (Date S1), with Christensenellaceae_R-7_group, Rikenellaceae_RC9_gut_group, 189 and Ruminococcaceae_NK4A214_group being dominant. Among them, the relative abundance of 190 Christensenellaceae_R-7_group was significantly lower in the $\mathrm{G}+\mathrm{S}$ group than in the G group 191 ( $P<0.05)$, while the relative abundance of Rikenellaceae_RC9_gut_group was significantly 192 higher $(P<0.05)$.

\section{Metagenomic functional profile predictions of the microbial communities}

194 PICRUSt software predicted the gene families related to the microbes identified by 16S rRNA 195 gene sequencing, and 43 KEGG gene families and 25 COG gene families were identified (Date 196 S2). Among the 43 KEGG gene families, >60\% were associated with metabolism, with the 197 largest proportion being related to carbohydrate metabolism. The function of carbohydrate 198 metabolism was significantly less common in the $\mathrm{G}+\mathrm{S}$ group than in the G group $(P<0.05)$ (Fig. 199 2). However, the function of glycan biosynthesis and metabolism was significantly more 200 common in the G+S group than in the G group. Second, Global and Overview Maps, Amino acid 201 metabolism and Energy metabolism accounted for the largest proportion, and the functions of 202 Amino acid metabolism and Energy metabolism were significantly higher in the G+S group than 203 in the $G$ group. Among the 25 COG gene families, transcription was the most frequent. In 204 addition, the most abundant functional categories of Carbohydrate transport and metabolism, 205 Amino acid transport and metabolism, and Energy production and conversion were significantly 206 different between the two groups of cattle-yak. Carbohydrate transport and Amino acid transport 207 and metabolism were significantly higher in the $G$ group than in the $G+S$ group. However, 208 Energy production and conversion was significantly lower in the $G$ group than in the $G+S$ group, 209 which is consistent with the KEGG functional analysis.

\section{Rumen VFAs and ruminal epithelial SGLT1 expression}

211 The total concentration of VFAs was significantly higher in the $G+S$ group than in the $G$ group $212(P<0.01)$. Acetate, propionate, butyrate, and isobutyrate concentrations were also significantly 213 higher in the $\mathrm{G}+\mathrm{S}$ group than in the $\mathrm{G}$ group $(P<0.01)$, but there were no significant differences 214 between the two groups in the other VFAs (valerate and isovalerate) $(P>0.05)$. Furthermore, the 
215 acetate/propionate ratio was significantly higher in the $\mathrm{G}+\mathrm{S}$ group than in the $\mathrm{G}$ group $(P<0.01)$ 216 (Table 3). Ruminal epithelial SGLT1 expression was significantly lower in the G+S group than in 217 the $\mathrm{G}$ group $(P<0.01)$. Correlation analysis revealed that VFAs had a definite association with the 218 SGLT1 gene; isobutyrate, butyrate, and valerate were significantly negatively correlated with 219 SGLT1 expression $(P<0.05)$, while isovalerate had a low correlation with SGLT1 expression (Fig. $2203)$.

\section{Discussion}

222 The rumen microbial community structure of ruminants is affected by numerous factors such as 223 feed variety, diet composition, seasonal changes, and feeding management (Yamano et al., 2019; 224 Li et al., 2019). The current study analyzed microbial communities in the rumen of cattle-yak 225 using 16S rRNA gene sequencing and revealed that supplementary feeding after grazing had little 226 effect on the $\alpha$-diversity index of microorganisms. This indicates that supplementary feeding after grazing did not change the microbial community diversity of the rumen, even though there were changes in the community composition and relative abundances of specific microorganisms. At the phylum level, Firmicutes, Bacteroidetes, and Actinobacteria were dominant in both groups of cattle-yak. Firmicutes possess many genes encoding energy metabolism-related enzymes that break down various substances and aid host digestion and absorption of nutrients (Kaakoush, 2015). Bacteroidetes can degrade carbohydrates and proteins (Nuriel-Ohayon et al., 2016; Fernando et al., 2010; Jami et al., 2014), and a high Firmicutes/Bacteroides ratio can help hosts effectively absorb energy-related nutrients and maintain their metabolic balance in lowtemperature environments (Ley et al., 2006; Fernando et al., 2010; Murphy et al., 2010). In the current study, cattle-yak in the $\mathrm{G}+\mathrm{S}$ group were affected by dietary composition (mainly roughage, supplemented with non-cellulose concentrate), which caused an increase in the content of Bacteroidetes and the ratio of Firmicutes/Bacteroides (Fernando et al., 2010), and consequently the $\mathrm{G}+\mathrm{S}$ cattle-yak could absorb energy-related nutrients more effectively and maintain their metabolic balance in the low-temperature environment (Murphy et al., 2010; Jami et al., 2014).

At the family level, there were some differences in the microbiota between the two groups of cattle-yak. Christensenellaceae and Ruminococcaceae, which are beneficial flora, were the predominant bacterial families in this study. Waters et al. (2019) found that Christensenellaceae is an important family of the phylum Firmicutes and is instrumental to human health. Rikenellaceae, a crucial polysaccharide-degrading family, displayed the highest abundance in the $\mathrm{G}+\mathrm{S}$ group. This may be related to the supplement feeding of concentrate, and by degrading more polysaccharides, extra energy is generated for the host (Laursen et al., 2017). At the genus level, Christensenellaceae_R-7_group belongs to the phylum Firmicutes (Waters and Ley, 2019) and mainly decomposes fibrous substances (Evans et al., 2011). Cattle-yak in the G group mainly fed on dry grass (high fiber), which may account for the highest relative abundance of Firmicutes and Christensenellaceae_R-7_group in this group. Rikenellaceae_RC9_gut_group is involved in degrading plant-derived polysaccharides (Seshadri et al., 2018), and its relative abundance increases with increased dietary fiber content (Qiu et al., 2019). In the current study, the relative abundance of Rikenellaceae_RC9_gut likely increased in the G+S group because cattle-yak in 
256 this group consumed more concentrate (mainly roughage with non-cellulose concentrate added). 257 Some studies have reported that Rikenellaceae_RC9_gut_group is related to the primary or 258 secondary degradation of carbohydrates (Pitta et al., 2010; Ramos et al., 2018). Therefore, 259 appropriate supplementary feeding after grazing in the cold season is speculated to promote 260 degradation of carbohydrates in the feed, which allow the cattle-yak to cope more effectively 261 with the harsh cold season.

262 PICRUSt software was used to predict microbial gene family functions. Cattle-yaks in G group 263 consumed some dried herbage with high fiber content, thus these animals need a large quantity of 264 microorganisms to degrade the carbohydrates with high cellulose content (Sun et al., 2015), and 265 this leads to enrichment of carbohydrate metabolism. In the KEGG analysis, the function of 266 glycan biosynthesis and metabolism was enriched in the G+S group. This was most likely 267 because cattle-yaks in the $\mathrm{G}+\mathrm{S}$ group consumed concentrate, which allows increased sugar 268 production. In addition, energy-related metabolic enrichment was found in both KEGG and COG 269 functional databases due to the addition of certain concentrates to meet specific energy 270 requirements in the G+S group. Concurrently, amino acid metabolism was enriched in the G+S 271 group. This suggests that after supplementation with certain concentrates, amino acid metabolism 272 might also provide some energy for the host.

273 Research has demonstrated that $>70 \%$ of VFAs are absorbed by the rumen epithelium, and these 274 are the main energy source for ruminants (Malmuthuge et al., 2015; Russell and Rychlik, 2001). 275 In this study, the G+S group of cattle-yaks had the highest concentrations of total VFA, acetate, 276 propionate, and butyrate. Supplementary feeding with an appropriate amount of concentrate 277 (which increases the soluble carbohydrate content of the diet) was previously shown to lead to more ruminal VFA production by microbial fermentation, thereby providing energy to the host (Qiu et al., 2019). In addition, a diet focused on cellulose reduced the total production of VFAs in the rumen, whereas feeding a certain amount of non-fibrous material increased propionate production (Polyorach et al., 2014; Liu et al., 2019). These findings are consistent with the results of the current study. The diet of cattle-yak in the G+S group was supplemented with noncellulose concentrate, resulting in increased propionate content, while cattle-yak in the G group had long-term intake of hay, which decreased the total VFA production. Furthermore, the acetate/ propionate ratio is correlated with feed energy use efficiency (Baldwin, 1998). This ratio was lower in cattle-yaks of group $G$ than those of group $G+S$ because group $G$ had long-term intake of forage with high fiber content, which enabled the cattle-yaks in this group to use energy more efficiently.

Nutrients are not only absorbed via the rumen epithelium in the form of VFAs; some glucose is directly absorbed by SGLT1 (Aschenbach et al., 2002; Aschenbach et al., 2000a). The relative energy associated with glucose rather than VFAs is much higher in ruminants (which rely almost entirely on gluconeogenesis to meet their glucose needs) than in monogastric species (Reynolds et al., 1994). Direct absorption of glucose via SGLT1 reduces the metabolic expenditure associated with gluconeogenesis in animals (Aschenbach et al., 2000b). In this study, expression of SGLT1 was lower in the G+S group than in the G group. A possible explanation is that to meet the energy needs of the cattle-yak in the G group, nutrients were absorbed in the form of VFAs 
297 and glucose was also directly absorbed via SGLT1 (Aschenbach et al., 2000a); this would aid the 298 cattle-yak in coping with the harsh cold season. Finally, correlation analysis demonstrated a 299 negative association of SGLT1 expression with VFAs. This indicated that ruminants absorb 300 energy in the form of VFAs but can also absorb glucose via SGLT1 to obtain energy. When VFA 301 concentrations decrease and energy supply is insufficient, SGLT1 gene expression increases and 302 energy is obtained directly by absorbing glucose, thus saving the energy required for 303 fermentation.

\section{Conclusions}

Intestinal microorganisms are an intrinsic part of the host lifecycle and influence animal phenotypes. Supplementary feeding in the cold season can improve the welfare of cattle-yak, which enhances production performance, disease resistance, and wintering ability. In this study, the rumen microbial flora of cattle-yaks exposed to two different grazing regimes were determined, and the interaction between cattle-yak rumen microbes and their metabolites and nutrient absorption-related genes of the host and their regulatory mechanisms were explored. Supplementary feeding in the cold season significantly increased the abundance of microbial flora and the VFA contents in the rumen of cattle-yak. There were also significant differences in the expression of SGLT1 in rumen epithelial tissue. Microbial function prediction revealed that carbohydrate metabolism and energy production functions were significantly enriched following supplementary feeding, and this interaction may play a specific regulatory role in the process of energy production. Therefore, supplementary feeding after returning to grazing in the cold season can help cattle-yaks better adapt to the harsh environment in the Qinghai-Tibet Plateau, which improves their wintering ability and welfare. Data from this study also provide a basis for future research on cattle-yak cold adaptation evolution in the Qinghai-Tibet Plateau.

\section{Supplementary Materials}

Date S1: Phylum, family, and genus horizontal species composition.

Date S2: Functional pathways of KEGG and COG genes.

Figure S1: (A) Venn diagram of OTU distribution; (B) rarefaction curves.

\section{ACKNOWLEDGMENTS}

We thank for the financial support: Basic Research Innovation Group Program of Gansu Province (17JR5RA137).

\section{References}


331

332

333

334

335

336

337

338

339

340

341

342

343

344

345

346

347

348

349

350

351

352

353

354

355

356

357

358

359

360

361

362

363

364

365

366
Aschenbach JR, Bhatia SK, Pfannkuche H, Gäbel G. 2000a. Glucose is absorbed in a sodiumdependent manner from forestomach contents of sheep. The Journal of Nutrition 130:27972801 DOI: 10.1093/jn/130.11.2797.

Aschenbach JR, Borau T, Gabel G. 2002. Glucose uptake via SGLT-1 is stimulated by beta(2)adrenoceptors in the ruminal epithelium of sheep. The Journal of Nutrition 132:1254-1257 DOI: $10.1093 /$ jn/132.6.1254.

Aschenbach JR, Wehning H, Kurze M, Schaberg E, Nieper H, Burckhardt G, Gäbel G. 2000b. Functional and molecular biological evidence of SGLT-1 in the ruminal epithelium of sheep. American Journal of Physiology-Gastrointestinal and Liver Physiology 279:20-27 DOI: 10.1152/ajpgi.2000.279.1.G20.

Baldwin RLT. 1998. Use of isolated ruminal epithelial cells in the study of rumen metabolism. The Journal of Nutrition 128:293S-296S DOI: 10.1093/jn/128.2.293S.

Evans NJ, Brown JM, Murray RD, Getty B, Birtles RJ, Hart CA, Carter SD. 2011. Characterization of novel bovine gastrointestinal tract treponema isolates and comparison with bovine digital dermatitis treponemes. Applied and Environmental Microbiology 77:138-147 DOI: 10.1128/AEM.00993-10.

Fernando SC, Purvis HTN, Najar FZ, Sukharnikov LO, Krehbiel CR, Nagaraja TG, Roe BA, Desilva U. 2010. Rumen microbial population dynamics during adaptation to a high-grain diet. Applied and Environmental Microbiology 76:7482-7490 DOI: 10.1128/AEM.0038810.

Jami E, White B.A, Mizrahi I. 2014. Potential role of the bovine rumen microbiome in modulating milk composition and feed efficiency. PLoS One 9: e85423 DOI: 10.1371/journal.pone.0085423.

Jing X, Peng Q, Hu R, Wang H, Yu X, Degen A, Zou H, Bao S, Zhao S, Wang Z. 2017. Effect of supplements during the cold season on the reproductive system in prepubertal Tibetan sheep ewes. Animal Science Journal 88:1269-1278 DOI: 10.1111/asj.12762.

Jing XP, Peng QH, Hu R, Zou HW, Wang HZ, Yu XQ, Zhou JW, Allan D, Wang ZS. 2018. Dietary supplements during the cold season increase rumen microbial abundance and improve rumen epithelium development in Tibetan sheep. Animal Science Journal 96:293305 DOI: 10.1093/jas/skx032.

Kaakoush NO. 2015. Insights into the role of Erysipelotrichaceae in the human host. Frontiers in Cellular and Infection Microbiology 5:84 DOI: 10.3389/fcimb.2015.00084.

Laursen MF, Bahl MI, Michaelsen KF, Licht TR. 2017. First foods and gut microbes. Frontiers in Microbiology 8:356 DOI: 10.3389/fmicb.2017.00356

Ley RE, Turnbaugh PJ, Klein S, Gordon JI. 2006. Microbial ecology: human gut microbes associated with obesity. Nature 444:1022-1023 DOI: 10.1038/4441022a. 
367

368

369

370

371

372

373

374

375

376

377

378

379

380

381

382

383

384

385

386

387

388

Li F, Li C, Chen Y, Liu J, Guan LL. 2019. Host genetics influence the rumen microbiota and heritable rumen microbial features associate with feed efficiency in cattle. Microbiome 7:92 DOI: 10.1186/s40168-019-0699-1.

Lin L, Xie F, Sun D, Liu J, Zhu W, Mao S. 2019. Ruminal microbiome-host crosstalk stimulates the development of the ruminal epithelium in a lamb model. Microbiome 7:83 DOI: 10.1186/s40168-019-0701-y.

Liu H, Xu T, Xu S, Ma L, Han X, Wang X, Zhang X, Hu L, Zhao N, Chen Y, Pi L, Zhao X. 2019. Effect of dietary concentrate to forage ratio on growth performance, rumen fermentation and bacterial diversity of Tibetan sheep under barn feeding on the QinghaiTibetan plateau. PeerJ 7:e74622 DOI: 10.7717/peerj.7462.

Liu X, Sha Y, Dingkao R, Zhang W, Lv W, Wei H, Shi H, Hu J, Wang J, Li S, Hao Z, Luo Y. 2020. Interactions between rumen microbes, VFAs, and host genes regulate nutrient absorption and epithelial barrier function during cold season nutritional stress in Tibetan sheep. Frontiers in Microbiology 11:593062 DOI: 10.3389/fmicb.2020.593062.

Livak KJ, Schmittgen TD. 2001. Analysis of relative gene expression data using real-time quantitative PCR and the 2- $\Delta \Delta \mathrm{CT}$ method. Methods 25:402-408 DOI: 10.1006/meth.2001.1262.

Malmuthuge N, Griebel PJ, Guan LL. 2015. The gut microbiome and its potential role in the development and function of newborn calf gastrointestinal tract. Frontiers in Veterinary Science 2:36 DOI: 10.3389/fvets.2015.00036.

Malmuthuge N, Guan LL. 2017. Understanding host-microbial interactions in rumen: searching the best opportunity for microbiota manipulation. Journal of Animal Science and Biotechnology 8:8 DOI: 10.1186/s40104-016-0135-3.

Murphy EF, Cotter PD, Healy S, Marques TM, O'Sullivan O, Fouhy F, Clarke SF, O'Toole PW, Quigley EM, Stanton C, Ross PR, O'Doherty RM, Shanahan F. 2010. Composition and energy harvesting capacity of the gut microbiota: relationship to diet, obesity and time in mouse models. Gut 59:1635-1642 DOI: 10.1136/gut.2010.215665.

Nagaraja TG. Microbiology of the Rumen. In: Millen D, De Beni Arrigoni M, Lauritano Pacheco RD, editors. Rumenology. 1st ed. Springer; Cham, Switzerland: 2016. pp. 39-62.

Nuriel-Ohayon M, Neuman H, Koren, O. 2016. Microbial changes during pregnancy, birth, and infancy. Frontiers in Microbiology 7:1031 DOI: 10.3389/fmicb.2016.01031.

Pitta DW, Pinchak WE, Dowd SE, Osterstock J, Gontcharova V, Youn E, Dorton K, Yoon I, Min BR, Fulford JD. 2010. Rumen bacterial diversity dynamics associated with changing from bermudagrass hay to grazed winter wheat diets. Microbial Ecology 59:511-522 DOI: 10.1007/s00248-009-9609-6.

Polyorach S, Wanapat M, Cherdthong A. 2014. Influence of yeast fermented cassava chip protein (YEFECAP) and roughage to concentrate ratio on ruminal fermentation and microorganisms

PeerJ reviewing PDF | (2020:08:52441:2:0:NEW 1 Feb 2021) 
404

using in vitro gas production technique. Asian-Australasian Journal of Animal Sciences 27:36-45 DOI: 10.5713/ajas.2013.13298.

Qiu Q, Zhu Y, Qiu X, Gao C, Wang J, Wang H, He Y, Rahman MAU, Cao B, Su H. 2019. Dynamic variations in fecal bacterial community and fermentation profile of Holstein steers in response to three stepwise density diets. Animals (Basel) 9:560 DOI: 10.3390/ani9080560.

Ramos AFO, Terry SA, Holman DB, Breves G, Pereira LGR, Silva AGM, Chaves AV. 2018. Tucumã Oil Shifted Ruminal Fermentation, Reducing Methane Production and Altering the Microbiome but Decreased Substrate Digestibility Within a RUSITEC Fed a Mixed Hay Concentrate Diet. Frontiers of Microbiology 9:1647 DOI: 10.3389/fmicb.2018.01647.

Reynolds CK, Harmon DL, Cecava MJ. 1994. Absorption and delivery of nutrients for milk protein synthesis by portal-drained viscera. Journal of Dairy Science 77:2787-2808 DOI: 10.3168/jds.S0022-0302(94)77220-9.

Russell JB, Rychlik JL. 2001. Factors that alter rumen microbial ecology. Science (New York, N.Y.) 292:1119-1122 DOI: 10.1126/science.1058830.

Segata N, Izard J, Waldron L, Gevers D, Miropolsky L, Garrett WS, Huttenhower C. 2011. Metagenomic biomarker discovery and explanation. Genome Biology 12:R60 DOI: 10.1186/ gb-2011-12-6-r60.

Seshadri R, Leahy SC, Attwood GT, Teh KH, Lambie SC, Cookson AL, Eloe-Fadrosh EA, Pavlopoulos GA, Hadjithomas M, Varghese NJ, Paez-Espino D, Perry R, Henderson G, Creevey CJ, Terrapon N, Lapebie P, Drula E, Lombard V, Rubin E, Kyrpides NC, Henrissat B, Woyke T, Ivanova NN, Kelly WJ. 2018. Cultivation and sequencing of rumen microbiome members from the Hungate1000 Collection. Nature Biotechnology 36:359-367 DOI: $10.1038 /$ nbt.4110.

Sun B, Wang X, Bernstein S, Huffman MA, Xia D, Gu Z, Chen R, Sheeran LK, Wagner RS, Li J. 2016. Marked variation between winter and spring gut microbiota in free-ranging Tibetan Macaques (Macaca thibetana). Scientific Reports 6:26035 DOI: 10.1038/srep26035.

Sun Y, Angerer J, Hou F. 2015. Effects of grazing systems on herbage mass and liveweight gain of Tibetan sheep in Eastern Qinghai-Tibetan Plateau, China. The Rangeland Journal 37:181190 DOI $=10.1071 / \mathrm{RJ} 14062$.

Wang Y, Sheng H, He Y, Wu J, Jiang Y, Tam NF, Zhou H. 2012. Comparison of the levels of bacterial diversity in freshwater, intertidal wetland, and marine sediments by using millions of illumina tags. Applied and Environmental Microbiology 78:8264-8271 DOI: 10.1128/AEM.01821-12.

Waters JL, Ley RE. 2019. The human gut bacteria Christensenellaceae are widespread, heritable, and associated with health. BMC Biology 17:83 DOI: 10.1186/s12915-019-0699-4. 
440 White JR, Nagarajan N, Pop M. 2009. Statistical methods for detecting differentially abundant 441 features in clinical metagenomic samples. PLoS Computational Biology 5:e1000352 DOI: $442 \quad$ 10.1371/journal.pcbi.1000352.

443 Wiener G, Han J, Long R. 2011. The Yak. Rap. Publication. 44:57-58 DOI: EP1087057A3.

444 Xin GS, Long RJ, Guo XS, Irvine J, Ding LM, Ding LL, Shang ZH. 2011. Blood mineral status 445 of grazing Tibetan sheep in the Northeast of the Qinghai-Tibetan Plateau. Livestock ence 446 136:102-107 DOI: 10.1016/j.livsci.2010.08.007.

447 Yamano H, Ichimura Y, Sawabe Y, Koike S, Suzuki Y, Kobayashi Y. 2019. Seasonal differences 448 in rumen bacterial flora of wild Hokkaido sika deer and partial characterization of an 449 unknown bacterial group possibly involved in fiber digestion in winter. Animal Science $450 \quad$ Journal 90:790-798 DOI: 10.1111/asj.13203.

451 Yohe TT, Schramm H, White RR, Hanigan MD, Daniels KM. 2019. Form of calf diet and the 452 rumen. II: Impact on volatile fatty acid absorption. Journal of Dairy Science 102:8502-8512 453 DOI: $10.3168 /$ jds.2019-16450.

454 Yu J, Cai L, Zhang J, Yang A, Wang Y, Zhang L, Guan LL, Qi D. 2020. Effects of thymol 455 supplementation on goat rumen fermentation and rumen microbiota in vitro. 456 Microorganisms 8:1160 DOI: 10.3390/microorganisms8081160. 


\section{Table 1 (on next page)}

Primer sequences for mRNA expression analysis.

SGLT1: sodium ion-dependent glucose transporter 1 
Table 1: Primer sequences for mRNA expression analysis.

\begin{tabular}{|c|c|c|c|c|}
\hline \multirow{3}{*}{ Gene } & \multirow{3}{*}{ Primer sequence $\left(5^{\prime}-3^{\prime}\right)$} & Length & & GenBank accession \\
\hline & & \multicolumn{3}{|c|}{ Temperature $\left({ }^{\circ} \mathrm{C}\right)$} \\
\hline & & (bp) & & number \\
\hline \multirow{2}{*}{ SGLT1 } & F: GTTTGCCTATGGAACCGGGA & \multirow{2}{*}{148} & \multirow{2}{*}{60} & \multirow{2}{*}{ NM_174606.2 } \\
\hline & R: TGCAATGGGCTTGGTGAAGA & & & \\
\hline \multirow{2}{*}{$\beta$-actin } & F: CGCAAGTACTCCGTGTGGAT & \multirow{2}{*}{146} & \multirow{2}{*}{60} & \multirow{2}{*}{ NM_173979.3 } \\
\hline & R: TAACGCAGCTAACAGTCCGC & & & \\
\hline
\end{tabular}




\section{Figure 1}

PICRUSt functional predictions based on the Kyoto Encyclopedia of Genes and Genomes (KEGG) and Clusters of Orthologous Groups (COG) databases.

(A) KEGG database; (B) COG databases. Bars on the left indicate the abundance ratio for each function in the two sets of samples; the graph on the right shows the percentage of difference in functional abundance within the $95 \%$ confidence interval; and $P$ values are displayed on the far right of each panel. G: grazing; G+S: grazing + supplementary feeding. 


\section{$\square \mathrm{G}+\mathrm{S} \square \mathrm{G}$}

Immune diseases

Metabolism of cofactors and vitamins

Energy metabolism

Nucleotide metabolism

Biosynthesis of other secondary metabolites

Amino acid metabolism

Xenobiotics biodegradation and metabolism

Metabolism of other amino acids $E$

Glycan biosynthesis and metabolism

Global and overview maps

Drug resistance $\mathrm{B}$

Membrane transport

Cell motility

Carbohydrate metabolism

Excretory system

Replication and repair

Cell growth and death $\mathrm{B}$

Transport and catabolism B

Infectious diseases: Parasitic |

Environmental adaptation

Substance dependence

Endocrine and metabolic diseases

Neurodegenerative diseases

Digestive system

Lipid metabolism

Folding, sorting and degradation

Nervous system

Cancers: Overview

Transcription

Immune system

Infectious diseases: Viral

Metabolism of terpenoids and polyketides

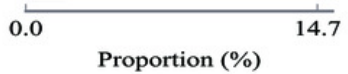

Proportion (\%)
Translation

Signal transduction

$95 \%$ confidence intervals

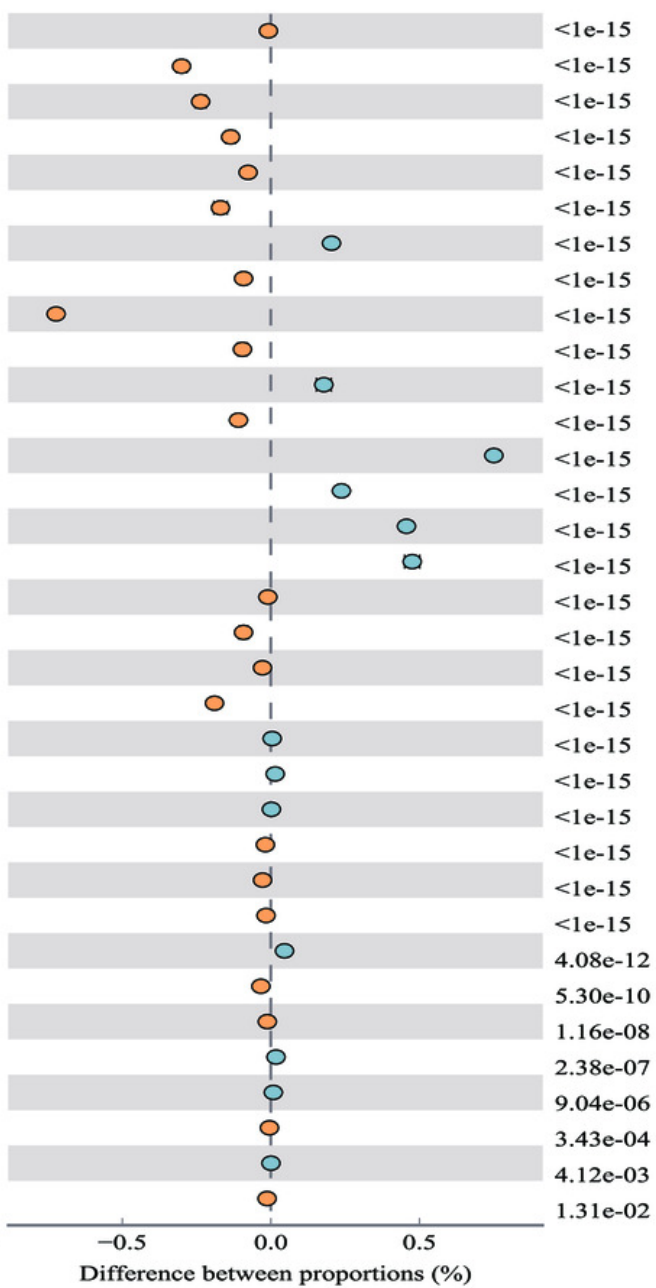

$95 \%$ confidence intervals

B

Cytoskeleton

Energy production and conversion Amino acid transport and metabolism Nucleotide transport and metabolism Carbohydrate transport and metabolism Coenzyme transport and metabolism Lipid transport and metabolism Translation, ribosomal structure and biogenesis Cell wall/membrane/envelope biogenesis Transcription Posttranslational modification, protein turnover, chaperones Inorganic ion transport and metabolism Function unknown Signal transduction mechanisms Intracellular trafficking, secretion, and vesicular transport Cell motility

RNA processing and modification Secondary metabolites biosynthesis, transport and catabolism Defense mechanisms

Chromatin structure and dynamics

Cell cycle control, cell division, chromosome partitioning

0.0

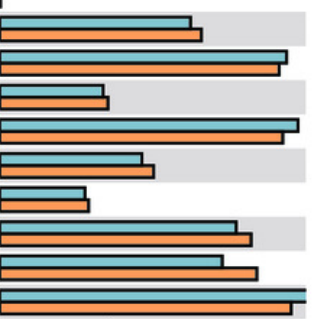

글

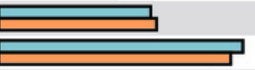

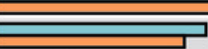

?

政

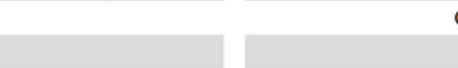

0

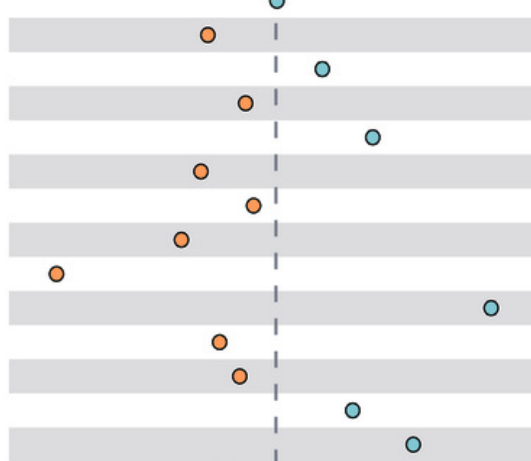

$<1 \mathrm{e}-15$

$<1 \mathrm{e}-15$

$<1 \mathrm{e}-15$

$<1 \mathrm{e}-15$

$<1 \mathrm{e}-15$

$<1 \mathrm{e}-15$

$<1 \mathrm{e}-15$

$<1 \mathrm{e}-15$

$<1 \mathrm{e}-15$

$<1 \mathrm{e}-15$

$<1 \mathrm{e}-15$

$<1 \mathrm{e}-15$

$<1 \mathrm{e}-15$ हे

$<1 \mathrm{e}-15$

$<$ e-15

$<1 \mathrm{e}-15$

$<1 \mathrm{e}-15$

$1.32 \mathrm{e}-12$

7.93e-09

$1.53 \mathrm{e}-08$

$1.37 \mathrm{e}-05$ 


\section{Table 2 (on next page)}

Effect of supplementary feeding after grazing on diversity index.

G: grazing; G+S: grazing + supplementary feeding; Chaol and Ace indices measure species abundance; Shannon and Simpson indices measure species diversity; Coverage index represents OTU coverage. 
1 Table 2: Effect of supplementary feeding after grazing on diversity index.

\begin{tabular}{lcccccc}
\hline Group & Number of OTUs & ACE & Chao1 & Simpson & Shannon & Coverage \\
\hline $\mathrm{G}+\mathrm{S}$ & 498 & 501.85 & 505.06 & 0.04 & 4.33 & 0.10 \\
$\mathrm{G}$ & 499 & 500.16 & 500.07 & 0.05 & 4.49 & 0.10 \\
& & & & & & \\
P value & 0.53 & 0.48 & 0.69 & 0.51 & 0.51 & 0.69 \\
\hline
\end{tabular}

2 G: grazing; G+S: grazing + supplementary feeding; Chaol and Ace indices measure species abundance; Shannon

3 and Simpson indices measure species diversity; Coverage index represents OTU coverage.

4 


\section{Table 3 (on next page)}

Effects of supplementary feeding after grazing on rumen fermentation parameters and SGLT1 gene expression.

G: grazing; G+S: grazing + supplementary feeding; SGLT1: sodium ion-dependent glucose transporter 1; VFA: volatile fatty acid. 
1 Table 3: Effects of supplementary feeding after grazing on rumen fermentation parameters and SGLT1 gene

2 expression.

\begin{tabular}{|c|c|c|c|}
\hline Ruminal VFAs (mmol/L) & $\mathbf{G}+\mathbf{S}$ & $\mathbf{G}$ & $P$ \\
\hline Acetate & $47.97 \pm 3.26$ & $36.75 \pm 0.39$ & 0.004 \\
\hline Propionate & $17.94 \pm 0.71$ & $15.47 \pm 0.09$ & 0.004 \\
\hline Butyrate & $7.30 \pm 0.00$ & $6.46 \pm 0.10$ & 0.000 \\
\hline Isobutyrate & $1.41 \pm 0.03$ & $1.32 \pm 0.02$ & 0.011 \\
\hline Isovalerate & $2.44 \pm 0.14$ & $2.53 \pm 0.07$ & 0.406 \\
\hline Valerate & $1.47 \pm 0.11$ & $1.29 \pm 0.04$ & 0.064 \\
\hline Total VFA & $78.54 \pm 3.68$ & $63.82 \pm 0.71$ & 0.002 \\
\hline Acetate/propionate ratio & $2.67 \pm 0.08$ & $2.38 \pm 0.01$ & 0.003 \\
\hline \multicolumn{4}{|l|}{ Gene expression } \\
\hline SGLT1 & $1.08 \pm 0.10$ & $5.56 \pm 0.61$ & 0.005 \\
\hline
\end{tabular}

3 G: grazing; G+S: grazing + supplementary feeding; SGLT1: sodium ion-dependent glucose transporter 1; VFA:

4 volatile fatty acid. 


\section{Figure 2}

Rumen microbial species composition of yak-cattle in grazing group and supplementary feeding group under different taxonomic classification levels.

(A) Phylum; (B) Family; (C) Genus. Each bar represents the average relative abundance of each bacterial taxon within a group. G: grazing; G+S: grazing + supplementary feeding.

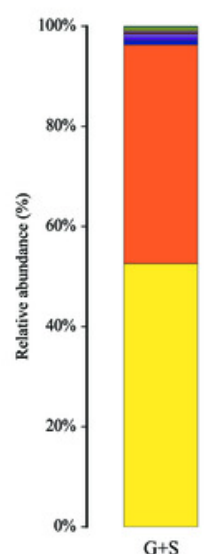

$\mathrm{G}+\mathrm{S}$

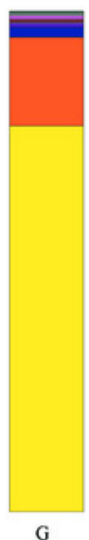

A

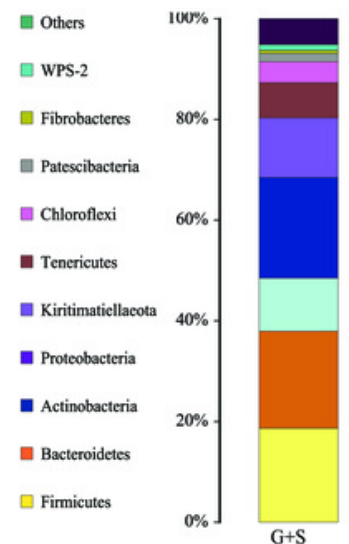

B
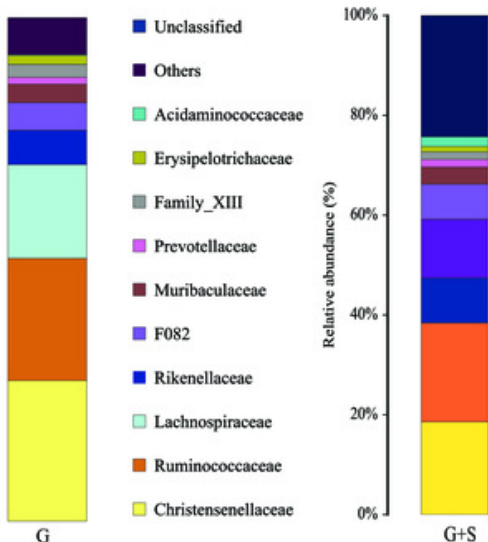

$\mathrm{G}+\mathrm{S}$

C

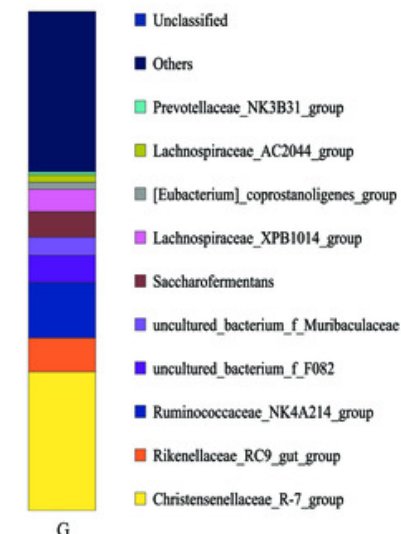


Figure 3

Correlation matrix between VFAs and SGLT1 gene.

Asterisks indicate significance of correlation at the $0.05\left(^{*}\right)$ and $0.01(* *)$ levels (both twotailed). SGLT1: sodium ion-dependent glucose transporter 1 . 


\begin{tabular}{|c|c|c|c|c|c|c|c|}
\hline & Acetate & Propionate & Isobutyrate & Butyrate & Isovalerate & Valerate & SGLT1 \\
\hline Acetate & 1.000 & $1.000 * *$ & 0.771 & 0.771 & -0.486 & 0.771 & -0.771 \\
\hline Propionate & $1.000 * *$ & 1.000 & 0.771 & 0.771 & -0.486 & 0.771 & -0.771 \\
\hline Isobutyrate & 0.771 & 0.771 & 1.000 & $1.000 * *$ & -0.029 & $1.000 * *$ & $-0.886 * *$ \\
\hline Butyrate & 0.771 & 0.771 & $1.000 * *$ & 1.000 & -0.029 & $1.000 * *$ & $-0.886 * *$ \\
\hline Isovalerate & -0.486 & -0.486 & -0.029 & -0.029 & 1.000 & -0.029 & 0.257 \\
\hline Valerate & 0.771 & 0.771 & $1.000 * *$ & $1.000 * *$ & -0.029 & 1.000 & $-0.886 * *$ \\
\hline SGLT1 & -0.771 & -0.771 & $-0.886^{* *}$ & $-0.886 * *$ & 0.257 & $-0.886 * *$ & 1.000 \\
\hline
\end{tabular}

